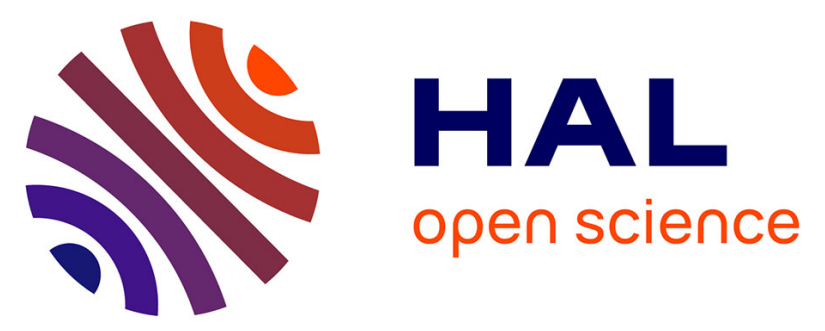

\title{
A fast 3D semi-analytical model for simulating flaw responses provided by a magnetic flux leakage NDT system inspecting ferromagnetic pipes
}

\author{
A. Trillon, F. Deneuville, D. Prémel, S. Djafa, Lionel Pichon
}

\section{To cite this version:}

A. Trillon, F. Deneuville, D. Prémel, S. Djafa, Lionel Pichon. A fast 3D semi-analytical model for simulating flaw responses provided by a magnetic flux leakage NDT system inspecting ferromagnetic pipes. QNDE 2013 - 40TH Annual Review of Progress in Quantitative Nondestructive Evaluation, Jul 2013, Baltimore, MD, United States. pp.1421-1427, 10.1063/1.4864988 cea-01820688

\section{HAL Id: cea-01820688 \\ https://hal-cea.archives-ouvertes.fr/cea-01820688}

Submitted on 22 Jul 2020

HAL is a multi-disciplinary open access archive for the deposit and dissemination of scientific research documents, whether they are published or not. The documents may come from teaching and research institutions in France or abroad, or from public or private research centers.
L'archive ouverte pluridisciplinaire HAL, est destinée au dépôt et à la diffusion de documents scientifiques de niveau recherche, publiés ou non, émanant des établissements d'enseignement et de recherche français ou étrangers, des laboratoires publics ou privés. 


\section{A fast 3D semi-analytical model for simulating flaw responses provided by a magnetic flux leakage NDT system inspecting ferromagnetic pipes}

Cite as: AIP Conference Proceedings 1581, 1421 (2014); https://doi.org/10.1063/1.4864988 Published Online: 17 February 2015

Adrien Trillon, François Deneuville, Denis Prémel, Steve Djafa, and Lionel Pichon

\section{ARTICLES YOU MAY BE INTERESTED IN}

Magnetic mechanisms of magnetic flux leakage nondestructive testing Applied Physics Letters 103, 184104 (2013); https://doi.org/10.1063/1.4828556

Simulation of magnetic flux leakage: Application to tube inspection AIP Conference Proceedings 1430, 1918 (2012); https://doi.org/10.1063/1.4716445

Pulsed magnetic flux leakage method for hairline crack detection and characterization AIP Advances 8, 047207 (2018); https://doi.org/10.1063/1.4994187
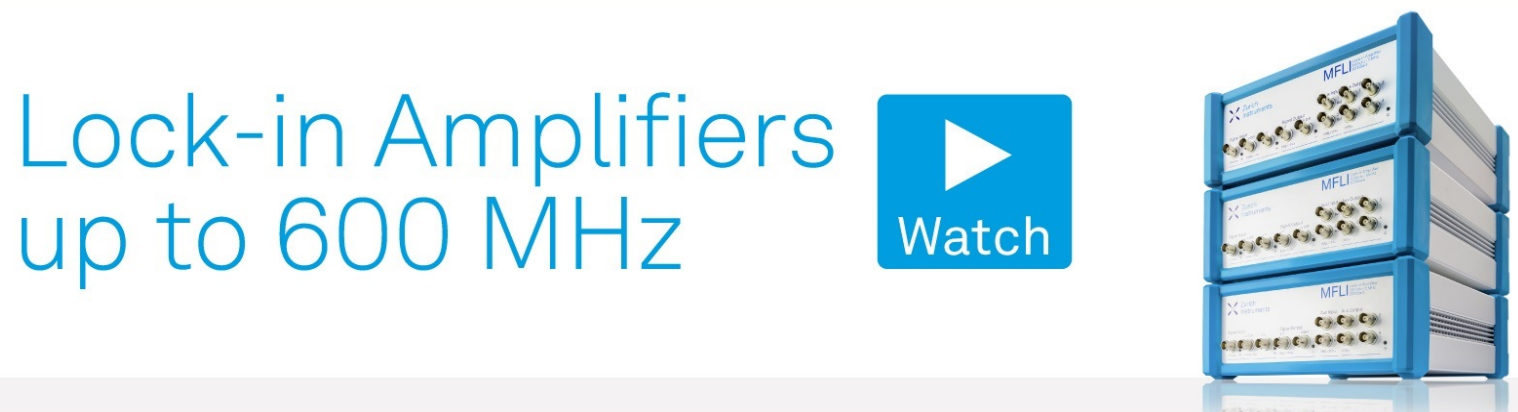


\title{
A Fast 3D Semi-Analytical Model for Simulating Flaw Responses Provided by a Magnetic Flux Leakage NDT System Inspecting Ferromagnetic Pipes
}

\author{
Adrien Trillon ${ }^{\mathrm{a}}$, François Deneuville ${ }^{\mathrm{a}}$, Denis Prémel $^{\mathrm{b}}$, Steve Djafa ${ }^{\mathrm{b}}$ \\ and Lionel Pichon ${ }^{\mathrm{c}}$ \\ ${ }^{a}$ Vallourec Research Aulnoye, 60 route de Leval, 59620 Aulnoye-Aymeries, France \\ ${ }^{b}$ CEA-LIST, Centre de Saclay, 91191 Gif-sur-Yvette, France \\ ${ }^{c}$ Laboratoire de Génie Electrique de Paris (LGEP), Supelec, 91191 Gif-sur-Yvette, France
}

\begin{abstract}
This communication presents a semi-analytical model based on BEM formalism for computing MFL flaw responses. MFL 2D configurations have been already simulated in the linear case taking into account the complex shape of the magnetizing circuit surrounding the pipe to be inspected. In this paper, the 3D problem is considered, but, in a first step, in order to be able to solve the non-linear problem, we assume that each ferromagnetic part can be divided in a finite number of polyhedral cells of complex shapes, assuming that the relative magnetic permeability is constant inside each cell. The nonlinear magnetosattic problem may be tackled as a finite number of successive 3D linear problems: the unknowns of the problem being the values of the surface magnetic charge density, in this case, the scalar potential of single layer charge on all the facets of each cell. By assuming that the unknowns are piecewise constant on all the facets of each cell, the kernels derived from integral equations can be analytically evaluated. The implemented semi-analytical model reveals itself to be very effective. Some simulated data are presented for some specific configurations before solving truly NDT MFL configurations.
\end{abstract}

Keywords: Eddy current testing, Nondestructive testing, modeling.

PACS: 02.60.Nm, 07.05.Tp, 07.55.Ge.

\section{INTRODUCTION}

In the quality control loop of pipe production, Nondestructive Testing (NDT) devices are crucial. Among the different existing techniques (Ultrasonic Testing (UT), Eddy Current Testing (ECT), Radiography Testing (RT), etc.), Magnetic Flux Leakage (MFL) testing is known to be efficient to ensure the detection of volume defects in ferromagnetic pipes with a low wall thickness.

Magnetic flux leakage testing is based on the flowing of a magnetic field through the wall of the pipe. Since the pipe is ferromagnetic, the pipe channels the magnetic field. In case of defect, the magnetic field leaks from the pipe [1]. On the outer side of the pipe, the magnetic field is modified. This modification of the magnetic field can be measured through inductive coils or Hall effect sensors. The principle of the MFL testing is comparable to Magnetic Particle Inspection (MPI) classically resorted to detect defects on the outer side of the object under test. MPI is also based on the magnetization of the piece but leaks are revealed through magnetic particles which agglomerate around the defect.

The improvement of MFL testing can be performed through new sensors or new processing of the raw signals. To help in the development of sensors and processing, VRA has invested in a lab bench illustrated Figure 1.

This kind of bench is very useful and efficient to improve knowledge and enhance sensors and processing. However, before time consuming and costly trials on a real bench, it is easier to perform modeling. To our knowledge there is no software dedicated to MFL testing modeling like CIVA for UT, ET and RT. The only way to simulate MFL testing is to resort to finite element models with software able to handle electromagnetic equations. However, finite elements are known to be efficient to simulate problems with complex geometries. Nevertheless, even with commercial software the models must be designed and the computation may be very time-consuming. To overcome this problem, a model is proposed to quickly simulate magnetic field in the case of MFL testing on pipes.

40th Annual Review of Progress in Quantitative Nondestructive Evaluation AIP Conf. Proc. 1581, 1421-1427 (2014); doi: 10.1063/1.4864988 C) 2014 AIP Publishing LLC 978-7354-1211-8/\$30.00 


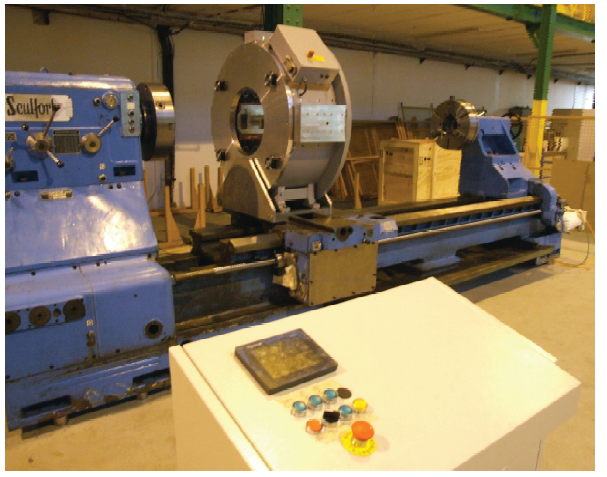

(a) Global overview

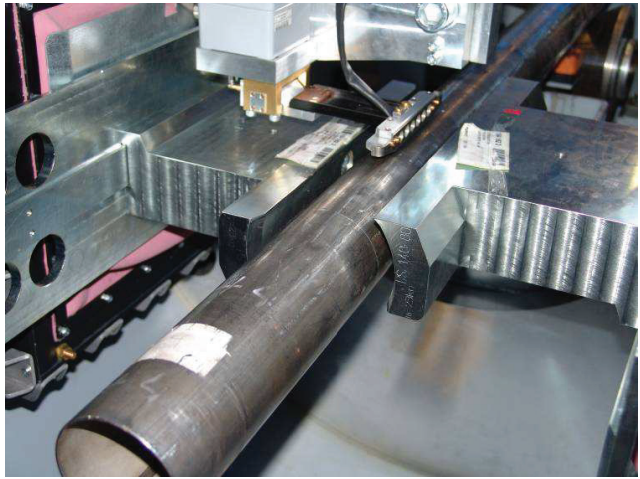

(b) Pipe and pole shoes

FIGURE 1. VRA lab bench for magnetic flux leakage testing.

A first model has been proposed [2], however it is limited to 2D geometries and nonlinear cases. The nonlinear assumption allows to take into account the hysteresis loop of the ferromagnetic material. However the 2D assumption limits the simulations to infinite lengthwise defect or infinite benches. This assumption prevents from modeling realistic cases. The proposed model hereafter is developed to simulate 3D geometries. In a first development, the medium is assumed piecewise linear.

\section{PROPOSED 3D MODEL FOR MFL TESTING}

The goal is to build up a 3D model to simulate the magnetic flux leakage testing. In order to obtain a fast and accurate 3D numerical model, we choose to use a BIM formalism and a scalar auxiliary potential function. By using the simple layer BIE formulation, one can obtain, in the non-linear case, a couple of integral equations implying the surface magnetic charge density $\sigma$ and the volume magnetic charge density $\rho$. This formulation is described in [2]. These auxiliary functions are related to the magnetizing vector:

$$
\sigma(\mathbf{r})=\mathbf{M}(\mathbf{r}) \cdot \mathbf{n}(\mathbf{r}) \text { and } \rho(\mathbf{r})=-\nabla \cdot \mathbf{M}=-\boldsymbol{H}(\boldsymbol{r}) \cdot \nabla \ln \left(\mu_{r}(\boldsymbol{r})\right)
$$

Where $\mu_{r}(\boldsymbol{r})$ stands for the relative magnetic permeability of the material. Since this formulation may be provide inaccurate results for materials of high permeability, another formulation is derived by considering a perturbation approach with introduce in a previous step the $\boldsymbol{H}_{\infty}$ field calculated with an infinite permeability. [3, 4, 5]. Though this formulation is very useful to solve various non-linear magnetostatic configurations, we adopted another strategy based on the moment magnetic method [6,7]. This approach consists to divide the ferromagnetic domain in a finite number of cells and let us assuming that the relative magnetic permeability $\mu_{r}$ is constant in each cell. Therefore, the volume magnetic charge is cancelled in each cell and it is possible to carry back the nonlinear magnetostatic problem to a linear one, the unknowns of the problem being the values of the surface magnetic charge density $\sigma_{i}$ on each facets $i$ of each cell. In our case, we consider $N_{F}$ facets of a polyhedral which can be a cell of complex shape.

To solve the problem is divided into a finite number of cells. These cells are polyhedra with complex shape. In each cell, the relative magnetic permeability $\mu_{r}$ is assumed constant. It does not mean that the relative magnetic permeability is necessary the same in each cell. The Boundary Integral formalism with the single layer potential formulation becomes:

$$
\begin{gathered}
\frac{1}{2} \sigma_{e, i}(\boldsymbol{r})+\lambda_{i}(\boldsymbol{r}) \sum_{e^{\prime}}^{N_{e}} \sum_{k}^{N_{f}} \int_{S_{k}} \sigma_{e^{\prime}, k}\left(\boldsymbol{r}^{\prime}\right) \partial_{n} G\left(\boldsymbol{r}, \boldsymbol{r}^{\prime}\right) \mathrm{d} s_{e^{\prime}, k}=-\lambda_{e, i}(\boldsymbol{r}) n(\boldsymbol{r}) \cdot \boldsymbol{H}_{0}(\boldsymbol{r}) \\
\boldsymbol{H}(\boldsymbol{r})=\boldsymbol{H}_{0}(\boldsymbol{r})+\sum_{e}^{N_{e}} \sum_{i}^{N_{f}} \int_{S_{i}} \sigma_{e, i}\left(\boldsymbol{r}^{\prime}\right) \nabla G\left(\boldsymbol{r}, \boldsymbol{r}^{\prime}\right) d s_{e, i}
\end{gathered}
$$


Where $\sigma_{e, i}(\boldsymbol{r})$ stands for the value of the surface charge density for the $i^{\text {th }}$ facets of the $e^{\text {th }}$ cell. Moreover:

$$
\nabla G\left(\boldsymbol{r}, \boldsymbol{r}^{\prime}\right)=-\frac{1}{4 \pi} \frac{\left(\boldsymbol{r}-\boldsymbol{r}^{\prime}\right)}{\left|\boldsymbol{r}-\boldsymbol{r}^{\prime}\right|^{3}} \text { and } \lambda_{e, i}(\boldsymbol{r})=\frac{\mu_{e, \text { inside }}-\mu_{e, \text { outside }}}{\mu_{1 e, \text { inside }}+\mu_{e, \text { outside }}}
$$

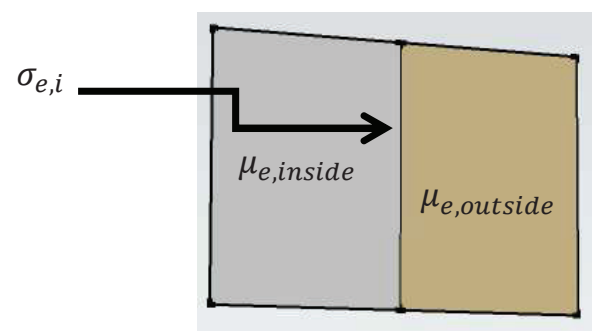

FIGURE 2. the relative magnetic permeability of two close cells.

If the relative magnetic permeability does not change between two close cell, the value of $\lambda_{e, i}(\boldsymbol{r})$ is null and the value of $\sigma_{e, i}(\boldsymbol{r})$ is also null. Otherwise, a new line of an algebraic matrix equation is introduced in order to evaluate of the values of the surface magnetic density for all cells. The advantage of this approach lies in the possibility to restrict the computation to the unknowns corresponding to the facets where there is a significant variation of the relative magnetic permeability between the two close cells.

In the linear case, one considers only the facets of the cells which are in contact with air, the difference of permeability between the cell and air being:

$$
\lambda_{e, i}(\boldsymbol{r})=\frac{\mu_{r}-1}{\mu_{r}+1}
$$

In the linear case, only magnetic densities on facets of the object under test in the air must be computed. In the nonlinear case, only facets presenting a difference of magnetic permeability must be assessed.

Finally, two ways are possible to discretize the integral equations through point matching method or Galerkin method. These two discretizing schemes have been implemented.

The followed algorithm is used to solve the non-linear problem with several steps:

1) Evaluate the initial permeability $\mu_{i}$;

2) Consider the facets of cells in contact with air;

3) Compute $\lambda_{k} \neq 0$ and solve the $3 \mathrm{D}$ linear case by computing the surface magnetic charge density on each facet of the cells;

4) Evaluate $H_{k}$ and the relative magnetic permeability $\mu\left(H_{k}\right)$ for each cell;

5) Update the relative magnetic permeability using a relaxation scheme;

6) Return to step 3 until convergence.

In order to validate the crucial step, i.e. solving the 3D linear case, the proposed model is firstly tested on three different configurations assuming that the relative permeability of the material is fixed to 100:

- 2 closed slabs;

- $\quad$ Tube with cylindrical coil;

- $\quad$ MFL bench with a simplified configuration.

\section{RESULTS}

Even if the approach is able to handle nonlinear media, a first step for any numerical validation of the approach has been carried out considering only linear medium. Three different cases are illustrated in Figure 2. 


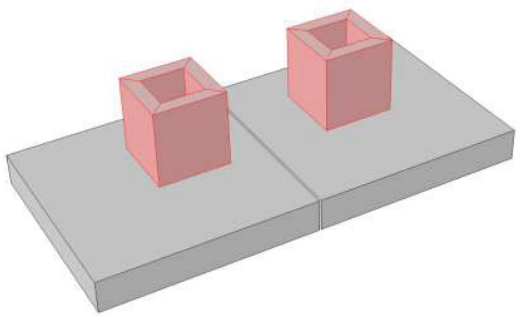

(a) Two closed slabs

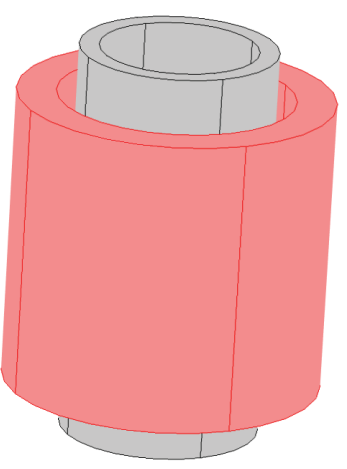

(b) Tube in cylindrical coils

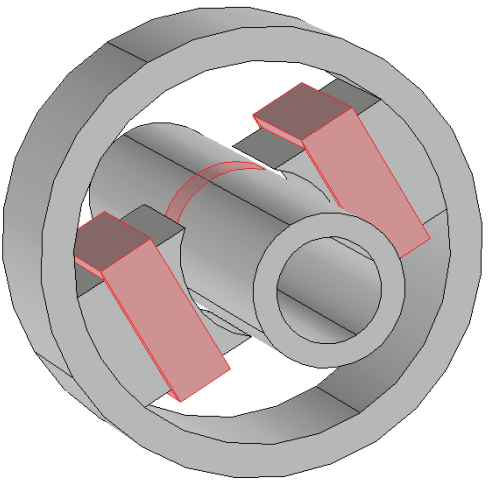

(c) MFL bench

FIGURE 2. Three tested cases.

The results of the model are compared with results obtained with a finite element model.

The first case is composed of two closed slabs with a gap of $0.826 \mathrm{~mm}$. Results for the first case are displayed Figure 3. On the left the results of the finite element models and on the right the results of the proposed model are displayed. A mapping z-component of the magnetic field on the plate and the modulus are displayed.

$\mathrm{Hz} \mathrm{EF}$

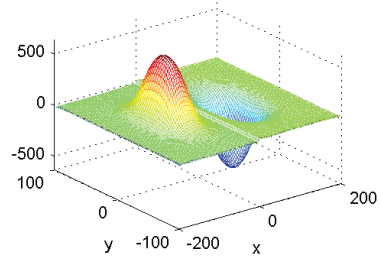

Module EF Hobs

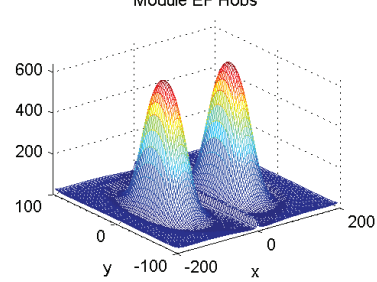

$\mathrm{Hz}$

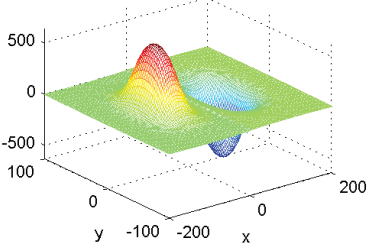

Hnorm

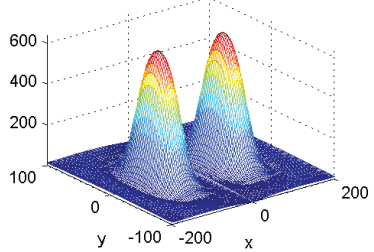

FIGURE 3. Results for the first case: comparison between the model and finite element.

Results of the two models are very close together. It shows the efficiency of the proposed approach in this paper. The two discretization methods (point matching and Galerkin methods) are compared together resorting to finite element results as reference on a line on the plate including the gap. The comparisons are displayed Figure 4. 

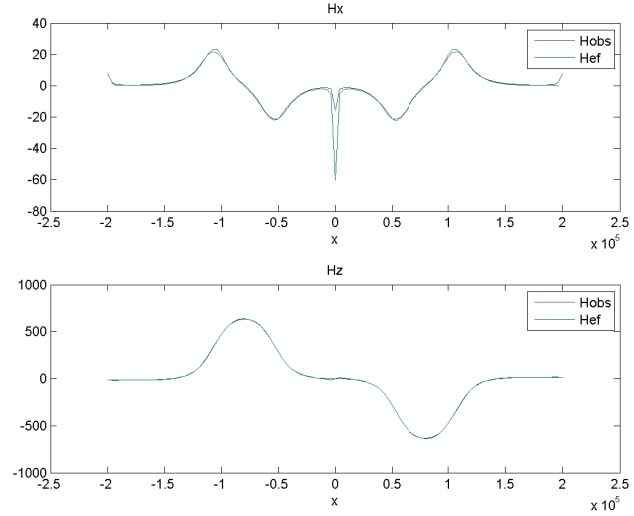

(a) Point matching method
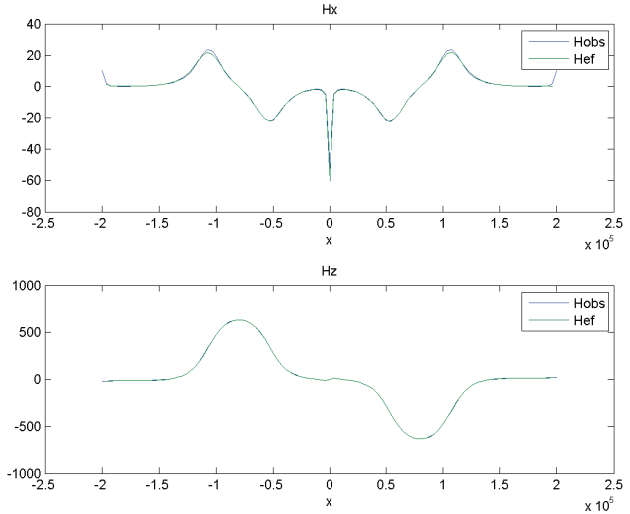

(b) Galerkin method

FIGURE 4. Comparison between point matching and Galerkin methods.

It appears that the two methods are efficient to discretize the semi-analytical model. However, it appears that the gap induces a difference in terms of accuracy: in middle of the gap the point matching method is less efficient that the Galerkin method. The latter is more accurate but takes much more time: few hours compared to the few minutes of the point matching method. For the next cases, only the point matching method is resorted due to a lower computation time.

The second case is composed of a pipe inserted in a cylindrical coil. The measurements of the magnetic field in plane through the coil and the pipe are illustrated Figure 5.
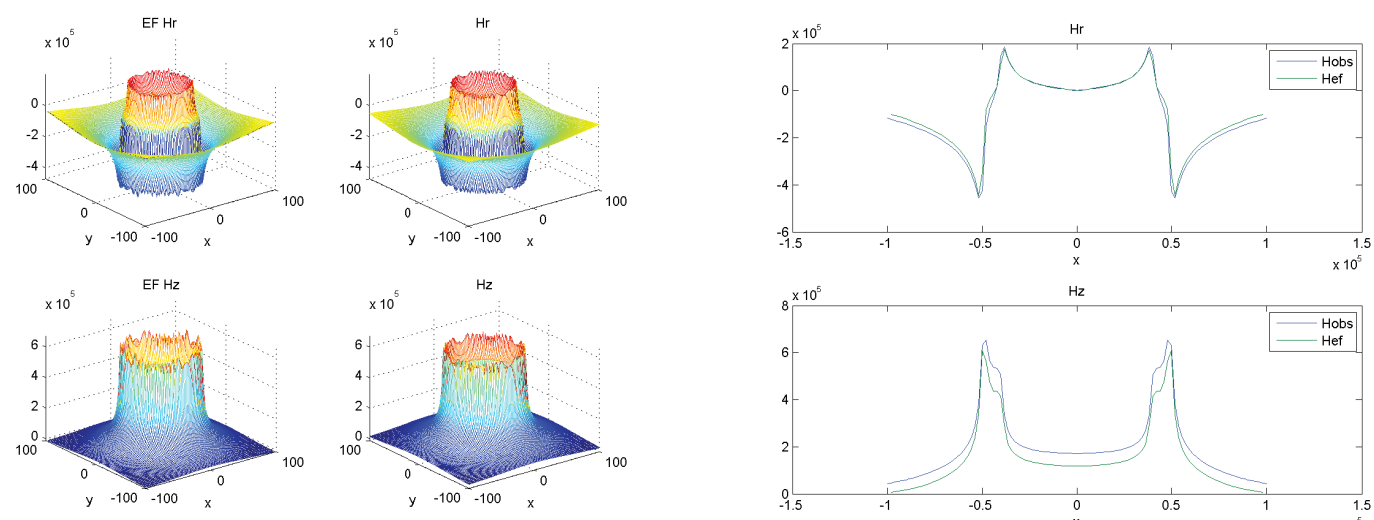

(a) Mapping of the magnetic field

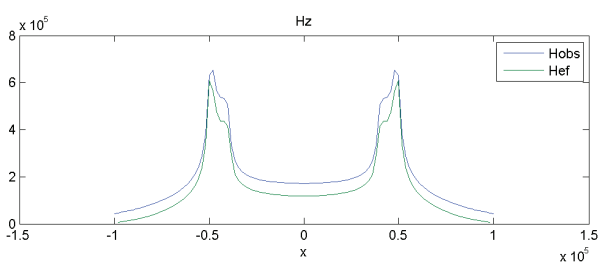

(b) Line of the magnetic field

FIGURE 5. Results for the second case: comparison between the model and finite element.

It appears that the results of the finite element method and the proposed approach are very close together illustrating the efficiency of the developed approach in this article.

The third case represents a simplified approach of the magnetic flux leakage bench available at the VRA. Results are displayed Figure 6. A comparison between finite elements and developed approach is performed. The results are presented with Cartesian and cylindrical coordinates.

The results between the two models are close together showing a high accuracy of the proposed approach. 

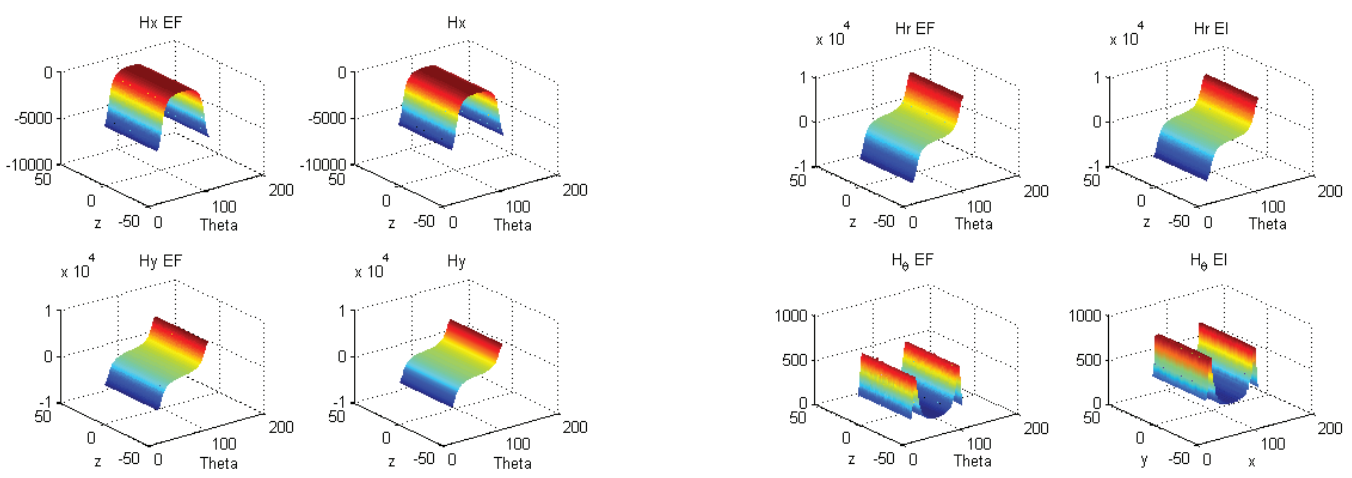

(a) Components of the magnetic field in a Cartesian coordinate system.
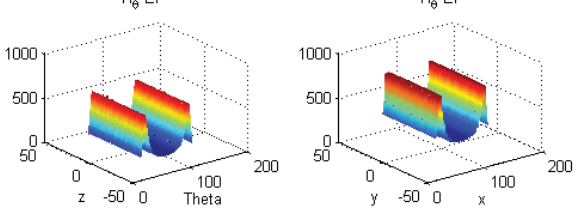

(b) Components of the magnetic field in a cylindrical coordinate system.

FIGURE 6. Results for the third case: comparison between the model and finite element.

\section{CONCLUSION}

The proposed approach shows a good matching with finite element methods in terms of simulated magnetic field in the case of linear medium. Further comparisons must be performed to validate the efficiency of the model for nonlinear medium.

The semi-analytical approach and the finite element approach have been compared and show close results. Nevertheless, the efficiency of these approaches with real measurements has not been assessed. To ensure the efficiency of the models, this comparison is important. It determines the reliability of the model.

To model the VRA bench, a large number of cells is necessary. It leads to large scale problems which are very difficult to solve. A possibility is to resort to Adaptive Cross Approximation (ACA) [8,9].

Nevertheless, the accuracy of the numerical model requires to be improved in order to be able to handle NDT geometries with flaws. To overcome this problem and to speed-up the model for the VRA lab bench and decrease the number of unknowns, it is possible to divide the problem into two steps:

- $\quad$ The first one with 3D modeling without any defect, the 3D global configuration is solved with a coarse mesh.

- $\quad$ The second one with a finite 3D modeling box surrounding the defect to model all possible geometries of defects. In this way, it is possible to have a finer mesh since we consider just a small box around the defect.

\section{REFERENCES}

1. Y. Sun, Y. Kang, "A new MFL principle and method based on near-zero background magnetic field", NDT \& $E$ International, 43, Issue 4, 348-353 (2010).

2. B. Krstajić, Z. andelić, S. Milojković and S. Babić, "Nonlinear 3D magetostatic field calculation by the ibntegral equation method with surface and volume magnetic charges", IEEE Trans. On Magnetics, 28 (2), 1088-1091 (1992).

3. W. Hafla, A. Buchau, F. Groh and and W.M. Rucker, "Fast Multipole Method Applied to Volume Integral Equation Method", Advances in Radio Science, 3, 195-198 (2005).

4. W. Hafla, A. Buchau and W.M. Rucker, "Accuracy improvement in nonlinear magnetostatic field computations with integral equation methods and indirect total scalar potential formulations", COMPEL - The International Journal for Computation and Mathematics in Electrical and Electronic Engineering, 25 (3), 565-571 (2006) .

5. D. Prémel, E.A. Fnaeich, S. Djafa, L. Pichon, A. Trillon and B. Bisiaux, "Simulation of magnetic flux leakage: Application to tube inspection", AIP Conference Proceedings, Thompson, D. O. \& Chimenti, D. E. (Eds.), AIP 1430, 1918-1925 (2012).

6. Y. Takahashi, C. Matsumoto and S. Wakao, "Large-Scale and Fast Nonlinear Magnetostatic Field Analysis by the Magnetic Moment Method With the Adaptive Cross Approximation”, IEEE Trans. on Magnetics, 43 (4), 1277-1280 (2007). 
7. O. Chadebec, J.L. Coulomb and F. Janet, “A review of magnetostatic moment method”, IEEE Trans. on Magnetics, 42 (4), 515-520 (2006).

8. Y. Takahashi, C. Matsumoto and S. Wakao, "Large-scale and Fast Nonlinear Magnetostatic Field Analysis by the Magnetic Moment Method with the Adaptive Cross Approximation", IEEE Trans. on Magnetics, 43 (4), 1277-1280 (2007).

9. V. Le Van, O. Chadebec, B. Bannwarth, A. Carpentier, J.M. Guichon and G. Meunier, "The Adaptive Cross Approximation Technique for the Integral Method in Magnetostatic”, EMF'2013 (2013). 